\title{
RUPTURA PREMATURA DE MEMBRANAS Y SU ASOCIACIÓN CON NEUMONÍA NEONATAL EN GESTANTES ATENDIDAS EN UN HOSPITAL TERCIARIO DE LIMA, PERÚ
}

\author{
Máryori Juárez-Peralta ${ }^{1, a}$, María Loo-Valverde ${ }^{1,2, b}$
}

\begin{abstract}
RESUMEN
Objetivo: Determinar la asociación entre ruptura prematura de membranas y neumonía en recién nacidos en un hospital terciario de Lima, Perú. Materiales y métodos: Basado en un estudio de cohorte retrospectiva, la selección de la muestra fue probabilístico aleatorio simple sin reposición, se obtuvo la muestra calculada para plantear grupos comparables y equilibrados. El tamaño de la muestra resultó en 102 gestantes de 102 gestantes, 51 con ruptura de membranas y 51 sin esta patología, en el hospital María Auxiliadora entre los años 2016 y 2017, se determinó la asociación de RPM y neumonía neonatal. Se utilizó el programa SPSS v.17 para un análisis descriptivo y cuadros de frecuencia utilizando un riesgo relativo con sus respectivos intervalos de confianza al 95\%. Resultados: En su totalidad, de las 102 gestantes de la muestra y sus respectivos productos, el $45 \%$ presentó neumonía y el $55 \%$ no presentó neumonía. Según el análisis, la frecuencia de neumonía neonatal fue mayor en los productos de aquellas gestantes que tenían RPM (58.8\%), con respecto a las que no tenían RPM (31.4\%) (p<0.00; RR: 2.340, IC95\%: 1.528 - 10.759) demostraron asociación significativa a RPM. Los exámenes de laboratorio y radiológicos patológicos también demostraron una asociación significativa. Conclusiones: El RPM está asociado significativamente a neumonía neonatal, con riesgo de 3 veces mayor en productos de gestantes con RPM respecto a las que no presentaron RPM.
\end{abstract}

Palabras claves: Ruptura prematura de membranas, neumonía neonatal, recién nacido (Fuente: DeCS BIREME).

\section{PREMATURE RUPTURE OF MEMBRANES AND ITS ASSOCIATION WITH NEONATAL PNEUMONIA IN PREGNANT WOMEN ATTENDED IN A TERTIARY HOSPITAL IN LIMA, PERU}

\begin{abstract}
Objective: To determine the association between premature rupture of membranes and pneumonia in newborns in a tertiary hospital in Lima, Peru. Materials and methods: Retrospective cohort study, the selection of the sample was simple random probability without replacement, the sample calculated to raise comparable and balanced groups was obtained. The sample size resulted in 102 pregnant women out of 102 pregnant women, 51 with ruptured membranes and 51 without this pathology, in the María Auxiliadora hospital between 2016 and 2017, the association of PMR and neonatal pneumonia was determined. The SPSS v.17 program was used for a descriptive analysis and frequency tables using a relative risk with their respective $95 \%$ confidence intervals. Results: In total, of the 102 pregnant women in the sample and their respective products, $45 \%$ had pneumonia and $55 \%$ had no pneumonia. According to the analysis, the frequency of neonatal pneumonia was higher in the products of those pregnant women who had RPM (58.8\%), compared to those who did not have RPM (31.4\%) $(p<0.00$; RR: $2,340,95 \% \mathrm{Cl}: 1,528-10,759)$ demonstrated significant association to RPM. Pathological laboratory and radiological examinations also demonstrated a significant association. Conclusions: The RPM is significantly associated with neonatal pneumonia, with a 3-fold increased risk in products from pregnant women with RPM compared to those who did not have RPM.
\end{abstract}

Key words: Premature rupture of membranes, neonatal pneumonia, newborn (Source: MeSH NLM).

\section{INTRODUCCIÓN}

La sepsis neonatal es un indicador que evidencia el nivel de desarrollo que tiene la atención prenatal y del recién nacido, en una determinada área geográfica o en un servicio. Si bien ésta patología ha disminuido en los últimos años, tanto en América Latina como en Perú, es un problema de salud que debe estudiarse para contribuir con nuevos aportes en poblaciones aún no estudiadas ${ }^{1}$.
Los primeros 28 días de vida son la etapa más vulnerable para la supervivencia del ser humano. En el mundo, ocurren alrededor de 3.9 a 10.8 millones de muertes anuales de menores de 28 días, la neumonía neonatal es la responsable de $20-38 \%$ durante las primeras 48 horas $^{2}$.

La mayoría de neumonías adquiridas por vía materna producen signos clínicos en los 3 primeros meses de vida y se calcula que la incidencia de neumonía precoz

Facultad de Medicina, Universidad Ricardo Palma. Lima, Perú

Hospital Edgardo Rebagliati Martins. Lima, Perú

Médica cirujana

Médica especialista en Pediatria, Doctora en Educación.

Citar como: Juárez-Peralta M, Loo-Valverde M. Ruptura prematura de membranas y su asociación con neumonía neonatal en gestantes atendidas en un hospital terciario de Lima, Perú Rev Peru Investig Matern Perinat 2020; 9(2): 16-20

DOI https://doi.org/10.33421/inmp.2020199

Recibido: 22-02-20 Aprobado: 30-06-20 
es del $0,5 \%$ en todos los recién nacidos vivos. En el Departamento de Pediatría de la Universidad Médica de Graz, Austria; se reportó frecuencias de neumonía neonatal en rangos de 1-35\%,1\% para los recién nacidos a término y el $10 \%$ para los recién nacidos prematuros. La incidencia varía según la edad gestacional, los criterios de diagnóstico o de definición de caso, el nivel y la calidad de la atención neonatal, la raza y el nivel socioeconómico ${ }^{3}$.

En el Instituto Nacional Materno Perinatal, el RPM se presentó con una frecuencia del $4-18 \%$ de los partos y se demostró que del $50 \%$ eran parto pretérmino y que contribuyó con el $20 \%$ de todas las muertes perinatales. En embarazos a término, se presentó en un $16-21 \%$ y en un embarazo pretérmino entre un $15-45 \%{ }^{4}$.

Se han realizado diversos estudios en neonatos con neumonía, en los cuales se presentan resultados positivos para gérmenes, como Staphylococcus aureus en mayor frecuencia, Escherichia coli, Klebsiella pneumoniae y Enterobacter cloacae. Generalmente, la neumonía neonatal es asociada a neonatos pretérminos, pero también se ha visto asociación con neonatos a término, con un tiempo de ingreso a hospitalización dentro de las 24 horas de vida y con peso al nacer normal ${ }^{4}$. Un estudio realizado en el Hospital Español de México sobre neumonía neonatal, mostró resultados en los que la mayoría de neonatos tuvieron Apgar 8 al primer minuto de vida, dichos neonatos nacieron por cesárea.

Dentro de los antecedentes perinatales maternos, se observó que el más frecuente fue la corioamnionitis en un $35 \%$, seguida de la ruptura prematura de membranas, la infección de vías urinarias, el cerclaje y la cervicovaginitis ${ }^{5}$.

En el presente estudio el objetivo fue determinar la asociación entre ruptura prematura de membranas y neumonía neonatal en gestantes atendidas en el hospital María Auxiliadora en el periodo Junio 2016-Junio 2017.

\section{MATERIALES Y MÉTODOS}

El tipo de investigación fue analítica, observacional, de cohortes retrospectivas. Participaron gestantes hospitalizadas en el Hospital María Auxiliadora en el periodo Junio 2016 a Junio 2017, que cumplan criterios de selección. Se formó el grupo de expuestos (gestantes con ruptura prematura de membranas) y grupo de no expuestos (gestantes sin ruptura de membranas).

Para el tamaño de muestro se empleó la fórmula para estudios de cohorte, considerando una diferencia de proporciones de dos grupos independientes con una hipótesis a dos colas, un $20 \%$ de pacientes expuestos, un poder estadístico de $80 \%$, un nivel de confianza del $95 \%$ y un riesgo relativo mayor de 1 , el tamaño de la muestra resultó de 51 gestantes con la exposición y 51 gestantes sin la exposición.
Criterios de inclusión para expuestos: a) gestantes con ruptura de membranas hospitalizadas, b) ocurrencia del parto en la institución. Criterio de inclusión para no expuestos: a) gestantes sin ruptura de membranas hospitalizadas, b) ocurrencia del parto en la institución. Criterios de exclusión para grupos de expuestos y no expuestos: Historia clínica con subregistro para las variables de estudio.

Variables consideras fueron edad materna indicada en años cumplidos, ruptura prematura de membranas indicando el tiempo en horas, neumonía neonatal indicada en registro médico, edad gestacional indicando si fue a término o pretermino, signos clínicos (tiraje, quejido respiratorio, taquipnea, disbalance toraco-abdominal, aleteo nasal), laboratorio (hemograma definido como patológico o a) y radiológicos registrados para el diagnóstico de neumonía neonatal en el registro médico.

La información que recogida en una ficha de recolección de datos ad hoc, a partir de la revisión de historia clínicas.

La información fue recolectada en una base de datos construida explícitamente para esta investigación, en el programa SPSS v. 17.0 y, a través del mismo, se obtuvieron los resultados del análisis de los datos mediante las funciones de análisis descriptivo y cuadros de frecuencia. Se generó una base de datos en Excel a la cual se pasó todos los datos obtenidos, el análisis estadístico se hizo con el programa SPSS y se hallaron los RR con sus respectivos intervalos de confianza al 95\%; así también, se representaron los resultados en tablas y figuras apropiados para su adecuada interpretación.

\section{RESULTADOS}

Se obtuvo una muestra de 102 neonatos, de los cuales se observa que el $45 \%$ presentó neumonía y el $55 \%$ no presentó neumonía (Tabla 1).

Tabla 1. Frecuencia de neumonía en neonatos

\begin{tabular}{ccc}
\hline Neumonia & $\mathrm{n}$ & Porcentaje \\
\hline $\mathrm{Si}$ & 46 & $45 \%$ \\
$\mathrm{No}$ & 56 & $55 \%$ \\
\hline
\end{tabular}

En relación a la edad materna, se observó que, dentro de las gestantes con RPM, la edad comprendida entre los 15 a 35 años es la que presentó mayor porcentaje en frecuencia (tabla 2).

Tabla 2. Estadística descriptiva de la edad materna en gestantes

\begin{tabular}{lcc}
\hline Edad materna & Si RPM & \multicolumn{2}{c}{ No RPM } \\
\hline menores de 15 años & $25 \%$ & $30 \%$ \\
$15-35$ años & $65 \%$ & $55 \%$ \\
mayores de 15 años & $10 \%$ & $15 \%$ \\
\hline
\end{tabular}


Con respecto a la frecuencia de dichas variables en los 102 neonatos, producto de ambas cohortes de gestantes, se observó que, los neonatos en poco porcentaje presentaron los signos clínicos, laboratoriales y radiológicos de neumonía neonatal descritos (Tabla 3 ).

Tabla 3. Frecuencia de signos clínicos, laboratoriales y radiológicos de neumonía en neonatos

\begin{tabular}{lrc}
\hline Clínica & $\mathbf{n}$ & porcentaje \\
\hline Tiraje & 26 & $25 \%$ \\
\hline Quejido respiratorio & 27 & $26 \%$ \\
\hline Taquipnea & 45 & $44 \%$ \\
\hline Disbalance toraco abdominal & 7 & $7 \%$ \\
Aleteo nasal & 9 & $9 \%$ \\
\hline Hemograma patológico & 26 & $25 \%$ \\
PCR elevado & 45 & $44 \%$ \\
Condensacion pulmonar & 45 & $44 \%$ \\
\hline
\end{tabular}

Respecto al grupo de gestantes con RPM, observamos que la edad gestacional promedio de la madre fue de 39 semanas y en la edad del neonato al diagnóstico se observó una edad promedio de 5 días. Respecto al grupo de gestantes sin RPM, la edad gestacional promedio fue 38 semanas y la edad del neonato al diagnóstico fue de 6 días. El tiempo de RPM promedio en las gestantes que lo presentaron fue de 18.50 horas.

Con respecto a la presentación de neumonía neonatal, la frecuencia fue mayor en los productos de aquellas gestantes que tenían RPM (58.8\%), con respecto a las que no tenían RPM (31.4\%). El intervalo de confianza al no contener la unidad nos confirma que el RPM es factor de riesgo para neumonía neonatal y el RR nos manifiesta que presenta 3 veces más riesgo de contraer la enfermedad en comparación a los productos de gestantes que no tienen RPM ( $p<0.05$, RR: 3.125, IC 95\%: 1.36-7.045) (Tabla 4).
Con respecto a la presentación de signos clínicos de neumonía neonatal, se observó que la frecuencia fue mayor en aquellos productos de gestantes que tenían RPM con respecto a las que no tenían no RPM. EI RPM demostró ser un factor de riesgo para presentar tiraje, quejido respiratorio y taquipnea en el neonato, debido a que cuentan con un $R R>1$, y la asociación es significativa debido a que el $p$ es $<0.05$. Respecto al disbalance tóraco abdominal y aleteo nasal, el RPM no demostró ser un factor de riesgo para presentar dichos signos clínicos (Tabla 4).

Con respecto a la presentación de signos laboratoriales patológicos, la frecuencia fue mayor en aquellos productos de gestantes que tenían RPM con respecto a las que no tenían RPM. El RPM demostró ser un factor de riesgo para presentar dichos signos en el neonato, debido a que cuentan con un $R R>1$, y la asociación es significativa debido a que el $p$ es $<0.05$ (Tabla 4).

Con respecto a la presentación de condensación pulmonar en radiografías, la frecuencia fue mayor en aquellos productos de gestantes que tenían RPM (56.9\%), con respecto a las que no tenían RPM (31.4\%). El intervalo de confianza muestra una asociación significativa por lo que el RPM está asociado con condensación pulmonar ( $p<0.01$, RR: 2.884, IC95\%: 1.282 - 6.485). Respecto a los signos radiológicos restantes como imágenes algodonosas, imágenes reticulares e imágenes nodulares, no se presentaron en ningún neonato de ambas cohortes de gestantes por lo que no hay asociación entre RPM y dichos signos radiológicos (Tabla 4).

\section{DISCUSIÓN}

La sepsis neonatal es una de las prioridades de salud a nivel nacional e internacional, dentro de la cual la neumonía neonatal es una patología muy frecuente. Este

Tabla 4. Asociación entre signos clínicos, laboratoriales y radiológicos de neumonía en neonatos y rotura prematura de membranas.

\begin{tabular}{|c|c|c|c|c|c|c|c|}
\hline & \multicolumn{3}{|c|}{ Con RPM } & \multicolumn{4}{|c|}{ Sin RPM } \\
\hline & $\mathbf{n}$ & Porcentaje & $\mathbf{n}$ & Porcentaje & $p$ & RR & IC95\% \\
\hline \multicolumn{8}{|l|}{ Diagnóstico de Neumonía } \\
\hline$-\mathrm{Si}$ & 30 & $58.80 \%$ & 16 & $31.40 \%$ & 0.005 & 3.13 & $1.36-1.05$ \\
\hline \multicolumn{8}{|l|}{ Clínica Respiratoria } \\
\hline - Tiraje & 21 & $41.20 \%$ & 5 & $9.80 \%$ & 0 & 6.44 & $2.19-18.93$ \\
\hline - Quejido respiratorio & 20 & $39.20 \%$ & 7 & $13.70 \%$ & 0 & 2.34 & $1.53-10.76$ \\
\hline - Taquipnea & 21 & $41.20 \%$ & 15 & $29.40 \%$ & 0 & 3.43 & $1.51-7.79$ \\
\hline - Disbalance toraco abdominal & 7 & $13.70 \%$ & 0 & $0.00 \%$ & 0.1 & 0.46 & $0.373-0.57$ \\
\hline - Aleteo nasal & 9 & $17.60 \%$ & 0 & $0.00 \%$ & 0.06 & 0.43 & $0.37-0.572$ \\
\hline \multicolumn{8}{|l|}{ Laboratorio } \\
\hline - Hemograma patológico & 19 & $37.30 \%$ & 7 & $13.70 \%$ & 0 & 2.77 & $1.4-9.93$ \\
\hline$-\mathrm{PCR}>0.5 \mathrm{mg} / \mathrm{L}$ & 29 & $56.90 \%$ & 16 & $31.40 \%$ & 2.88 & 2.88 & $1.3-6.48$ \\
\hline \multicolumn{8}{|l|}{ Signos Radiológicos } \\
\hline - Condensacion pulmonar & 29 & $56.90 \%$ & 16 & $31.40 \%$ & 0.01 & 2.88 & $1.28-6.48$ \\
\hline
\end{tabular}


origen corresponde tanto a la vida prenatal y al parto como a la vida posnatal, es por ello, por lo que deben observarse estrechamente los signos sugerentes de dificultad respiratoria en el neonato, con el fin de llevar a cabo un diagnóstico y un tratamiento precoz ${ }^{6}$.

Este estudio nos permitió conocer sobre el impacto de esta patología en el servicio de Neonatología del Hospital María Auxiliadora y su asociación con la ruptura prematura de membranas en las gestantes, el factor más comúnmente relacionado a neumonía neonatal según revisión de la bibliografía.

Se estableció como RPM a la pérdida de líquido mayor o igual a 12 horas y se estudió su asociación con neumonía neonatal mediante signos clínicos, radiológicos y de laboratorio, además de estadísticas descriptivas como la edad gestacional, la edad del recién nacido al diagnóstico y la edad materna. Se analizaron dichas variables en una muestra de 102 gestantes, siendo $50 \%$ las que presentaron RPM y $50 \%$ las que no presentaron RPM.

Se observó que la edad gestacional promedio fue de 38.46 semanas, con un intervalo de 37 - 40 semanas, mientras que la edad del recién nacido al diagnóstico fue de 5.47 días, con un intervalo de $1-13$ días. El tiempo promedio de RPM en las gestantes que lo presentaron fue de 18.50 horas. Vimos que, la neumonía neonatal también puede darse a gran escala en productos de gestantes a término, a diferencia de lo que plantea el estudio de Francisco Ortiz Maldonado ${ }^{7}$ sobre complicaciones neonatales asociadas a la ruptura prematura de membranas, que demostró que la proporción de neonatos con neumonía neonatal fue más alta en los menores de 35 semanas.

En relación a la variable de neumonía neonatal; se observa que, en total de ambas cohortes, el $45 \%$ de neonatos presentó neumonía y el $55 \%$ no presentó neumonía; sin embargo, sí se encuentra asociación significativa entre las gestantes con RPM y neumonía neonatal, con mayor frecuencia en los productos de aquellas gestantes que tenían RPM (58.8\%), con respecto a las que no tenían RPM (31.4\%) y con 3 veces mayor riesgo de presentarla. El intervalo de confianza al no contener la unidad, nos confirma que el RPM es factor de riesgo para neumonía neonatal y el RR, nos manifiesta que presenta 3 veces más riesgo de contraer la enfermedad en comparación a los productos de gestantes que no tienen RPM (p:0. 005, RR: 3.125, IC95\%: $1.386-7.045)$.

Según los antecedentes revisados, también se encuentran ahí asociación significativa entre éstas dos variables, como en el estudio de Timana $\mathrm{C}^{6}$ sobre factores asociados a riesgo para sepsis neonatal temprana, en el que se encuentra a RPM como factor de riesgo en un odds ratio de 3.34 y una incidencia de 5.08 x 100 recién nacidos.

Respecto a los signos clínicos de neumonía neonatal, se encontró una asociación significativa entre RPM y 3 categorías, excepto disbalance tóraco - abdominal y aleteo nasal, apoyando este estudio. Ningún producto de las gestantes sin RPM presentó disbalance tóraco abdominal ni aleteo nasal.

En relación a los signos de laboratorio de neumonía neonatal, considerando un hemograma patológico con leucocitosis más desviación izquierda y un valor de PCR $>0.5 \mathrm{mg} / \mathrm{L}$, se encontró asociación significativa con RPM. Otro estudio sobre características microbiológicas y terapéuticas de la sepsis neonatal, un $12.7 \%$ de neonatos presentó un hemograma patológico y el $14.5 \%$ presentó PCR elevado ${ }^{8}$.

Respecto a los signos radiológicos de neumonía neonatal, se encontró asociación significativa entre RPM y condensación pulmonar. Respecto a los signos radiológicos restantes como imágenes algodonosas, imágenes reticulares e imágenes nodulares, no se presentaron en ningún neonato de ambas cohortes de gestantes, por lo que no hay asociación entre RPM y dichos signos radiológicos.

Podemos concluir que, el tiempo de RPM en las gestantes, 18.50 horas en promedio, está asociado a neumonía neonatal. Los signos clínicos de neumonía neonatal están asociados al antecedente de RPM en la madre, a excepción de disbalance tóraco abdominal y aleteo nasal. Los signos de laboratorio patológicos también están asociados al antecedente de RPM en la madre. El único signo radiológico asociado significativamente a RPM es la condensación pulmonar. Se encontró asociación significativa entre RPM y neumonía neonatal.

Financiamiento: Autofinanciado.

Conflicto de interés: Los autores declaran no tener algún conflicto de intereses.

\section{REFERENCIAS BIBLIOGRÁFICAS}

1. Castro FW, Labarrere Y, González G, Barrios Y. Factores de riesgo del Síndrome Dificultad Respiratoria de origen pulmonar en el recién nacido. Rev Cubana Enfermer [Internet]. 2007 Sep [citado 2018 Jun 27]; 23(3). Disponible en: http://scielo.sld.cu/scielo.php?script=sci_arttext\&pid=S086403192007000300005\&lng=es.

2. Moya JKJ. Factores de riesgo de mortalidad neonatal temprana en el hospital Víctor lazarte Echegaray en el periodo 2011 - 2015, Trujillo, Perú. 2016. Disponible en: http://repositorio.upao.edu.pe/handle/upaorep/2158

3. Pozo J. Incidencia y factores de riesgo de la neumonía connatal en la Unidad de Cuidados Intensivos Neonatales del Hospital de Especialidades Guayaquil Dr. Abel Gilbert Pontón comprendido desde enero del 2013 a enero del 2015. Universidad de Guayaquil. Facultad de ciencias médicas. Escuela de medicina. 2015. Disponible en: http://repositorio. ug.edu.ec/handle/redug/10884

4. Flores JE. Factores de riesgo asociados a la ruptura prematura de membranas en embarazos pretérminos atendidos en 
el Instituto Nacional Materno Perinatal durante el periodo Enero-Diciembre, 2015. Universidad Nacional Mayor de San Marcos. Facultad de Medicina. 2016. Disponible en: https:// cybertesis.unmsm.edu. pe/handle/cybertesis/4979?show=full

5. Cabanillas-Carhuaz S. Características y complicaciones de gestantes con ruptura prematura de membranas pretérmino y a término. Rev Peru Obstet Enferm. 2015; 11 (2).

6. Timana C. Factores asociados a riesgo para sepsis neonatal temprana en el Hospital Nacional Hipólito Unanue de Lima Enero - Diciembre 2004. Universidad Ricardo Palma, facultad de Medicina. 2006. Disponible en: http://repositorio. urp.edu.pe/handle/urp/201

7. Ortiz-Maldonado F. Complicaciones neonatales asociadas a la ruptura prematura de membranas amnióticas en recién nacidos de pretérmino. Rev Mex Pediatr 2014; 81(5); 169173. Disponible en: https://www.medigraphic.com/pdfs/ pediat/sp-2014/sp145c.pdf
8. Cuipal JD. Características clínicas de la sepsis neonatal temprana en el Hospital Nacional Dos de Mayo, 2015. Universidad Nacional Mayor de San Marcos. Facultad de Medicina. 2016. Disponible en:

https://cybertesis.unmsm.edu.pe/handle/20.500.12672/4679

\section{Correspondencia:}

Máryori Juárez Peralta

Dirección: Av. Alfredo Benavides 5440, Santiago de Surco 15039 Teléfono: 986857824 